\title{
Gerenciamento de estoques via previsão de vendas agregadas utilizando simulação
}

\author{
Eduardo Carrara Peixoto \\ Belgo Bekaert Nordeste S.A. \\ Luiz Ricardo Pinto \\ UFMG
}

\begin{abstract}
Resumo
O aumento da economia global, a concorrência e a tecnologia exigem cada vez mais agilidade e flexibilidade das organizações. Na siderurgia isso não é diferente, apesar de seus processos requererem grandes escalas e conseqüente elevação dos tempos de lead time. A manutenção de estoques, nesse contexto, se torna imprescindível. Este trabalho apresenta uma contribuição para o processo de tomada de decisão para quantificação de estoques em uma empresa de trefilação de arames. Um modelo matemático foi elaborado para auxiliar as políticas de aquisição e manutenção de estoques de cada item. A empresa forneceu informações chave para o modelo: vendas agregadas de produtos acabados; demanda histórica de insumos; histórico de suprimentos; desempenho de fornecedores e estratégia de compra para o período. Como o sistema envolve variáveis estocásticas, fez-se necessário a determinação dos níveis de estoque de segurança para evitar rupturas na produção e no fornecimento de produto aos clientes.
\end{abstract}

Palavras-chave

Estoque, previsão de vendas, simulação, estoque de segurança, siderurgia.

\section{Inventory management based on forecast of aggregated sales using simulation}

\begin{abstract}
The increase of the global economy, the market competition and the technology demand more flexible and faster organizations. Despite the great production volume and its consequent increase of the lead time, the metallurgy industry is not out of this game. In this context, a good supply inventory policy may be a powerful tool. The article presents a contribution for the inventory decision process in a wire drawing industry. A descriptive mathematical model was built to support the supply order process of each product. The company supplied the key information of the model: aggregated sales of final products; forecast sales; supply items historical series; supplier's performance and order's strategy for the period. As the system involves stochastic variables, it was defined safety stocks to avoid production interruption and final products delivery interruption.
\end{abstract}

Key words

Inventory, forecast sales, simulation, safety stock, metallurgy. 


\section{INTRODUC̄̃̃O}

Certamente um dos passos mais importantes na condução de uma empresa é o planejamento. Analisar cenários e fazer a escolha do caminho por onde se deseja conduzir a empresa em longo prazo faz parte dessa etapa. Um bom planejamento é aquele que dita claramente quais mercados e clientes serão servidos e é baseado em uma visão da organização sobre como será o mercado no futuro e qual o papel da empresa nesse cenário. Por visão entende-se a definição de metas e ideais através dos quais os negócios serão conduzidos.

Decisões estratégicas, tais como aquelas que tratam de capacidade, nível de serviço e rede de distribuição, são complexas e envolvem o comprometimento de recursos ao longo de vários anos.

Em última instância, o que se busca através do planejamento empresarial é a identificação das necessidades do cliente e a adaptação dos processos de negócio para satisfazer essas necessidades.

Um importante desdobramento dessa atividade é o planejamento da produção. Um bom planejamento da produção não garante o sucesso, mas direciona os esforços da organização no sentido de maximizar a utilização de recursos e melhorar o desempenho total.

O planejamento da produção está ligado às políticas de manutenção de estoques. Os motivos pelos quais os estoques estão presentes nos canais de produção são vários, mas o planejamento dos níveis desses estoques está cada vez mais em evidência, pois eles se tornam variável determinante dos resultados financeiros de muitos ramos empresariais.

Lenard e Roy (1995) afirmam que o controle de estoque é estudado desde 1913 com Harris. Pode-se afirmar que o bom funcionamento de tal área é vital para uma organização, uma vez que representa boa parte dos custos logísticos. Assim, é evidente a necessidade de se buscar a otimização do gerenciamento desses serviços. Um bom gerenciamento de estoques equaciona as questões de disponibilidade, nível de serviço e custos de manutenção (KREVER et al., 2003).

Para Ballou (2001), os estoques estão presentes em um canal de suprimentos principalmente por: (i) melhorar o serviço ao cliente; (ii) reduzir os custos operacionais (set up, aquisição, transporte, etc.). No entanto, estoques são condenáveis devido a: (i) custo de sua manutenção; (ii) possibilidade de estoques poderem mascarar problemas de qualidade; (iii) obsolescência. Toda política de controle de estoques deve administrar o difícil dilema entre minimizar custos de manutenção de estoques e satisfazer a demanda do cliente.

Krupp (1997) afirma que, quanto maior o estoque, maior o nível de serviço. Krupp (2002) ressalta, no entanto, que em muitos ambientes a manutenção de estoques de produtos acabados ou semi-acabados não é permitida.
A partir da década de 70, surgiram vários sistemas de controle de produção (MRP, JIT e ERP) que têm características um pouco mais qualitativas em relação às abordagens quantitativas tradicionais.

Platt et al. (1997) destacam a existência de uma necessidade implícita nos modelos de gestão de estoques ligada a sua adequação e aplicabilidade à cadência normal das operações que podem implicar em freqüentes cálculos de tamanho de pedidos e ponto de ressuprimento (ROP) para centenas de itens ou produtos de uma empresa.

Hautaniemi e Pirttilä (1999) realizaram comparações entre algumas políticas de controle de estoques, tais como MRP, ROP e sistemas de revisão visuais como two-bin. Esses autores afirmam que, apesar dessas políticas serem consideradas mutuamente excludentes pela literatura, a necessidade de mercado impõe tempos de entrega cada vez menores, mais confiáveis e produtos personalizados, gerando a necessidade de usar ROP ou sistemas de revisão visuais juntamente com os sistemas MRP.

Os modelos científicos baseados em pesquisa operacional fornecem fundamentação teórica para a construção de sistemas de suporte à decisão na área de planejamento da produção e gerenciamento de estoques. As três principais abordagens voltadas para a modelagem computacional em pesquisa operacional baseiam-se em: otimização, heurística e simulação.

A otimização através de técnicas de programação matemática, tais como programações linear, não-linear e inteira, deve ser empregada sempre que possível, pois essas técnicas garantem uma solução ótima. A complexidade dos modelos, no entanto, normalmente demanda grande capacidade computacional e tempos de processamento proibitivos. Nesse caso, as heurísticas têm sido aplicadas para reduzir o espaço a ser pesquisado e posteriormente avaliar as alternativas encontradas através de uma das outras abordagens.

Simulação é um tipo de modelagem matemática que procura primeiramente retratar a dinâmica de um sistema existente ou planejado para depois poder avaliar soluções viáveis do problema. Essa técnica é recomendada quando se pretende abordar um nível de detalhe mais amplo do processo.

\section{DESCRICÃO DO SISTEMA ESTUDADO}

A empresa na qual foi desenvolvido este estudo faz parte do setor siderúrgico e está focada na produção de arames de aço para diversas aplicações industriais e comerciais. A empresa atua no mercado interno e externo e possui cinco unidades de produção. Seu principal insumo para produção é o fio máquina - FM. Fios máquina são fios de arame utilizados para fabricação de trefilados em geral. Atualmente são utilizados mais de seiscentos tipos de FM na fabricação 
de quase quatro mil itens de produtos acabados que a empresa oferece ao mercado. Esses números já dão uma idéia da complexidade do Planejamento e Controle da Produção PCP dessa empresa.

Para simplificar e viabilizar análises e verificar tendências, a empresa utiliza o monitoramento agregado de diversas variáveis do processo. Por agregação entende-se o agrupamento de diversos itens relacionados em grupos que passam então a representar um determinado conjunto de itens.

Assim, a área comercial faz seu planejamento avaliando setores de negócio ou grupos de produtos. Os chamados Grupos de Venda - GV tornam-se assim a base do planejamento de demanda (previsão de vendas) da empresa. Atualmente a empresa possui aproximadamente $120 \mathrm{GVs}$, cada qual constituído de diversos tipos de FM, aqui chamados FMItem. A demanda dos GVs e suas composições em termos de FMItem são variáveis aleatórias, cujos comportamentos históricos são conhecidos.

O desafio do presente trabalho foi definir corretamente as variáveis e parâmetros do processo decisório para retratá-lo num modelo matemático, baseado em conceitos de simulação de processos, que apóie a tomada de decisão de pedido de compra mensal do insumo FM.

O fornecedor do insumo FM é uma empresa do grupo que, visando planejar-se melhor e otimizar seus resultados, passou a exigir que os pedidos de FMItem fossem registrados no mês anterior a sua previsão de entrega.

\section{BASES METODOLÓGICAS DO TRABALHO}

A metodologia de pesquisa utilizada durante o desenvolvimento deste trabalho foi a de pesquisa-ação. A idéia central é usar uma abordagem científica para estudar a resolução de importantes matérias organizacionais juntamente com aqueles que vivenciam estas matérias diretamente (COUGHLAN; COGHLAN, 2002).

Na pesquisa-ação, membros do sistema que está sendo objeto de estudo participam ativamente, ao contrário da pesquisa tradicional, em que os membros do sistema são objetos do estudo. Ela também é simultânea com a ação. A meta é fazer uma ação efetiva enquanto simultaneamente se constrói um conhecimento científico. Todo o trabalho foi desenvolvido pelos autores com grande participação dos membros do corpo técnico da empresa. Este trabalho é uma síntese do trabalho de Peixoto (2006), cuja hipótese era que o comportamento histórico das previsões de venda de produtos acabados e o respectivo mix de produtos necessários à produção, adicionados a parâmetros e restrições de produção poderiam determinar, com boa precisão, o planejamento de compra de insumos. O desafio apresentado era que, utilizando os dados disponíveis de comportamento histórico da previsão de vendas agregadas de produtos acabados (GV) e o respectivo mix de insumos (FMItem), adicionados a parâmetros e restrições de produção, poder-se-ia determinar o planejamento de compra de cada item de fio-máquina para o mês seguinte ao planejamento. FMItem com a antecedência requerida, foi elaborado um modelo de avaliação dos dados históricos de desempenho desse processo para testar seu comportamento e distribuição. Também foram incluídas algumas informações relevantes a essa tomada de decisão.

Para as variáveis aleatórias desse processo foram associadas curvas de distribuição de probabilidade que, interagindo entre si, possibilitarão a definição do pedido de compra para cada FMItem através da utilização de técnicas de simulação.

Com base no estudo e tratamento dos dados coletados (histórico da movimentação de materiais entre a empresa e seus fornecedores do insumo FM) foi elaborado e implementado computacionalmente um modelo matemático. Esta ferramenta tem como objetivo gerar e avaliar cenários relacionados ao processo de compra e estocagem de FMItem.

$\mathrm{Na}$ definição de uma política de estoques, normalmente usa-se o critério de minimização do custo total. No entanto, para Hillier e Lieberman (2005), outro critério a ser considerado, embora não quantitativo, é que a política de estoque resultante seja simples. Por "simples" devemos entender a existência de uma regra clara para indicar quando e quanto encomendar.

Para Santos e Rodrigues (2005), algumas mudanças no mercado, como o crescimento do nível de personalização dos produtos, as fusões e aquisições no mercado industrial, geraram uma demanda extremamente irregular. Esse tipo de demanda é caracterizado por alto nível de variabilidade medido pelo coeficiente de variação (CV). Além disso, nesse tipo de demanda, há picos de consumo seguidos de vários períodos de consumo nulo ou baixo. 
O coeficiente de variação $(\mathrm{CV})$ mede a concentração, polarização ou amontoamento da demanda de um item (JACOBS e WHYBARK, 1992). A partir dele enxerga-se se a demanda do item é contínua ou intermitente. Porém, a definição do valor que caracteriza um CV como alto, médio ou baixo varia de um autor para outro. Ele é freqüentemente estimado como o resultado do desvio padrão da amostra dividido pela sua média. Pang et al. (2003) afirmam que mesmo para uma distribuição Normal, a exata distribuição do CV é difícil de ser obtida.

Segundo Petrovic e Petrovic (2001), os estoques operam em presença de eventos aleatórios. Portanto, diante de tantas variáveis que afetam o gerenciamento dos estoques, a definição da política de ressuprimento adequada é um problema desafiador.

a necessidade de simplificações do modelo real, mas ainda assim elas são ferramentas de aplicação computacional complexa, o que dificulta o seu uso prático.

É comum encontrar empresas que necessitam desenvolver políticas de estoques para centenas de itens. Clientes cada vez mais exigentes por diferentes produtos fazem com que o número de itens em estoque aumente consideravelmente. Isso fez com que o planejamento e o controle do estoque se tornassem uma atividade complexa, criando a necessidade de classificar os itens com multicritérios. Para Ramanathan (2004), esses critérios podem ser vários, tais como: custo de estoque, importância do item, tempo de produção, existência de atributos comuns, obsolescência, facilidade de substituição, número de reposições por ano, escassez, durabilidade, tamanho de lote de reposição, estocagem, distribuição de demanda e custo de penalidade por falta.

Segundo Partovi eAnandarajan (2002), quando há centenas de itens de estoque para controle, é comum se perder de vista o gerenciamento efetivo e adequado para cada tipo de material. Uma solução possível é a separação e a classificação dos itens em subgrupos, permitindo a adoção da melhor política para cada um desses.

Uma informação importante para apoiar o gestor de estoques durante sua tomada de decisão diz respeito à probabilidade de ruptura do estoque, ou seja, probabilidade de falta do produto no estoque para atender a demanda. De acordo com Yeh et al. (1997), a determinação da probabilidade de ruptura só pode ser feita após a determinação da distribuição de probabilidade que rege a amostragem da demanda.

Bartezzghi et al. (1999) propuseram um estudo, através do uso de simulação, para verificar quais os impactos das diferentes formas das distribuição de demanda nos estoques.

Podem-se encontrar na literatura diversos exemplos de políticas de gestão de estoques que, traduzidas matematicamente, foram posteriormente derivadas para que fosse possível determinar o ponto de ressuprimento, o lote econômico de aquisição, etc. Deve-se destacar, no entanto, que para chegar a equações matemáticas “tratáveis", as simplificações do modelo real são quase que uma imposição.

Platt et al. (1997) exemplificam essa prática afirmando que, na literatura, é muito comum observar a adoção da distribuição de demanda durante o lead time como normalmente distribuída. Em seu trabalho, eles utilizam essa simplificação. Ainda assim, a aplicação de métodos de solução exatos pode comprometer a aplicação prática dessa iniciativa. Custos elevados de desenvolvimento de modelos, dados variáveis e simplificações fora da realidade dos tomadores de decisão fazem com que os métodos aproximados dominem esse campo do conhecimento. As heurísticas reduzem
Assim a companhia opta por não despender grande esforço para determinar uma política ótima de estoque para cada item.

Para realização de um bom planejamento da produção é essencial que as empresas trabalhem com uma boa base nas suas previsões de venda. Previsões ruins vão levar a empresa a tomar decisões ruins, o que compromete a sua competitividade (CORRÊA, 2001).

No entanto, muito embora a previsão de demanda possa ser uma ferramenta importante no processo de planejamento para os gerentes, é difícil conseguir uma previsão totalmente correta. Ao contrário, na maioria dos casos são atingidos resultados muito distantes destes. Quanto maior for o horizonte de previsão, maiores serão os erros. Deve-se destacar ainda que a demanda possui algumas características que podem influenciar a previsão e as técnicas utilizadas para o seu cálculo (ARNOLD, 1999).

A palavra simulação tem diferentes significados dependendo do contexto em que está sendo aplicada. Nesse trabalho, simulação deve ser entendida como o processo de desenvolvimento de um modelo de um sistema real para condução de experimentos com o propósito de entender o funcionamento do sistema ou de avaliar estratégias de operação desse sistema (SHANNON, 1975).

A simulação não otimiza a solução do problema, mas permite escolher a melhor opção dentre as alternativas avaliadas. Uma de suas vantagens é a relativa simplicidade de 
construção de modelos se comparada à modelagem matemática tradicional (modelos de otimização). Outra vantagem é a possibilidade de utilização de variáveis probabilísticas ao longo de um período determinado.

A simulação pode ser utilizada fornecendo informações para dar suporte à tomada de decisão ou pode realmente fornecer uma solução (PRITSKER, 1992).

\section{DESCRIC̄ÃO DO MODELO}

Foi elaborado um modelo matemático representativo do sistema real, que foi implementado computacionalmente em Borland Delphi® usando base de dados em Microsoft Access ${ }^{\circledR}$.

As premissas do modelo são:

- periodicidade e revisão mensal do planejamento;

- restrições de volume de compra impostas pela "cota" (limite de volume) de suprimento do fornecedor de FM;

- aleatoriedade do erro cometido na previsão de vendas por GV e aleatoriedade na composição de cada GV (participação de cada FMItem na composição do GV).

Os cenários a serem avaliados pelo tomador de decisão são construídos a partir da variação dos parâmetros do nível de serviço e cotas de FMItem.

Na obtenção dos resultados a partir dos cenários construídos, o algoritmo do sistema opera da seguinte forma:

$1^{\circ}$ Passo: Determinação do estoque de segurança

$\mathrm{O}$ estoque de segurança ameniza variações e/ou incertezas de curto prazo, tanto de demanda quanto de ressupri- mento. No modelo desenvolvido, o estoque de segurança protege a produção das variações e incertezas do prazo de ressuprimento (lead time de entrega do fornecedor de fio máquina) e do histórico de consumo (oscilações no volume de demanda). Adicionalmente, permite gerar uma proteção maior com base no conceito de estoque de demanda média num "prazo de cobertura". Nesse último caso, o sistema atribui um valor adicional ao tradicional estoque de segurança para proteger a produção num prazo equivalente aos dias especificados. Isso é especialmente válido no caso de itens que têm cronograma de laminação muito espaçado.

Para indicar a confiabilidade dos estoques sugeridos, foram criados dois coeficientes de segurança para serem aplicados ao histórico: o de segurança na demanda (volume histórico) e o de segurança no tempo de ressuprimento (lead time do fornecedor). O processo é representado na Figura 1.

Vale ressaltar que na determinação dos históricos de demanda e atendimento, para garantir a representatividade dos dados, não se pode estender muito o horizonte analisado em função das mudanças do mercado e do processo.

Após alguns testes, avaliou-se que 12 meses é um horizonte reduzido, porém adequado nessa situação (amostra de dados reduzida compromete a determinação das curvas de distribuição de probabilidade que regem as variações / incertezas do processo de decisão). Os cálculos para a determinação do estoque de segurança são mostrados a seguir e, apesar das simplificações adotadas, mostraram-se bastante adequados para o problema em estudo.

Para cada fio máquina, calcula-se um valor para o lead time com base em dados históricos de atendimento dos pedi-

Figura 1: Diagrama de obtenção do estoque de segurança.

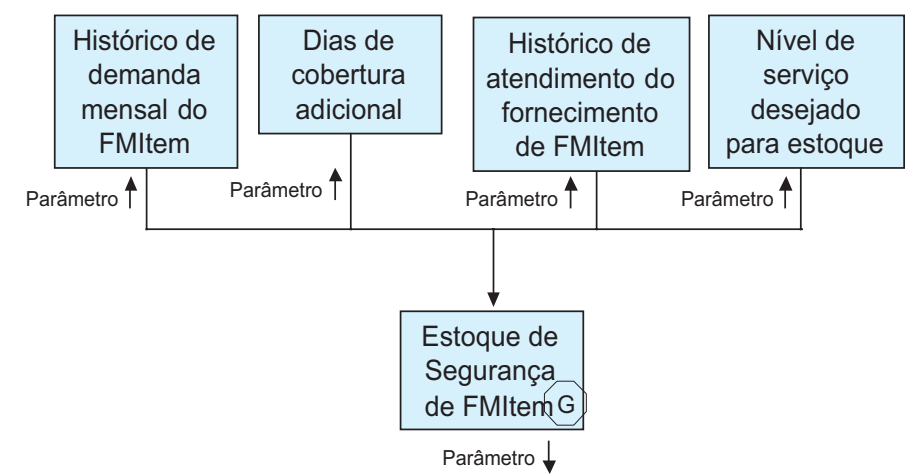

$\uparrow$ Entrada
$\downarrow$ Saída

Freqüência: Mensal 
dos de FMItem e obtém-se um conjunto de dados de prazos de atendimento para todos os FM.

$$
\mathrm{LT}_{\mathrm{i}}=\mathrm{LTM}_{\mathrm{i}}+\mathrm{DPL}_{\mathrm{i}} \cdot \mathrm{SL}_{\mathrm{i}}
$$

onde:

$\mathrm{LT}_{\mathrm{i}}=$ lead time previsto para o $\mathrm{FM}_{\mathrm{i}}$ (dias);

$\mathrm{LTM}_{\mathrm{i}}=$ lead time histórico médio para o $\mathrm{FM}_{\mathrm{i}}$ (dias);

$\mathrm{DPL}_{\mathrm{i}}=$ desvio padrão do lead time histórico para o $\mathrm{FM}_{\mathrm{i}}$ (dias);

$\mathrm{SL}_{\mathrm{i}}=$ fator de segurança para o lead time do $\mathrm{FM}_{\mathrm{i}}$ (valores de $\mathrm{SL}_{\mathrm{i}}$ no Quadro 1A).

A partir dos dados históricos de distribuição da demanda mensal de FM calcula-se a demanda de cada FMItem $\left(D_{i}\right)$ :

$\mathrm{D}_{\mathrm{i}}=\mathrm{DM}_{\mathrm{i}}+\mathrm{DPD}_{\mathrm{i}} \cdot \mathrm{SD}_{\mathrm{i}}$

onde:

$\mathrm{D}_{\mathrm{i}}=$ demanda mensal prevista para o $\mathrm{FM}_{\mathrm{i}}(\mathrm{t})$;

$\mathrm{DM}_{\mathrm{i}}=$ demanda mensal histórica média para o $\mathrm{FM}_{\mathrm{i}}(\mathrm{t})$; $\mathrm{DPD}_{\mathrm{i}}=$ desvio padrão da demanda mensal histórica para o $\mathrm{FM}_{\mathrm{i}}(\mathrm{t})$;

$\mathrm{SD}_{\mathrm{i}}=$ fator de segurança para a demanda do $\mathrm{FM}_{\mathrm{i}}$ (valores de $\mathrm{SD}_{\mathrm{i}}$ no Quadro 1A).

Para os cálculos de $\mathrm{D}_{\mathrm{i}}$ só foram considerados FMItem que aparecem no mínimo um determinado número de vezes (aqui chamado de repetitividade mínima) nos dados históricos e cujas demandas médias estejam acima de $25 \%$ do valor de seu desvio padrão. Este procedimento visa à eliminação de dados pontuais atípicos.
O estoque de segurança é calculado da seguinte forma:

$\mathrm{ES}_{\mathrm{i}}=\mathrm{EC}_{\mathrm{i}}+\mathrm{VD}_{\mathrm{i}}$
$\mathrm{EC}_{\mathrm{i}}=\mathrm{D}_{\mathrm{i}} \cdot \mathrm{DC}_{\mathrm{i}} / 30$
$\mathrm{VD}_{\mathrm{i}}=\mathrm{LT}_{\mathrm{i}} \cdot \mathrm{D}_{\mathrm{i}} / 30$
onde:
$\mathrm{ES}_{\mathrm{i}}=$ estoque de segurança para o $\mathrm{FM}_{\mathrm{i}} ;$
$\mathrm{EC}_{\mathrm{i}}=$ estoque de cobertura para o $\mathrm{FM}_{\mathrm{i}} ;$
$\mathrm{VD}_{\mathrm{i}}=$ valor esperado da demanda durante o período do
lead time para o FM;
$\mathrm{DC}_{\mathrm{i}}=$ número de dias desejáveis para cobertura da de-
$\mathrm{manda}_{\mathrm{i}}$ do $\mathrm{FM}_{\mathrm{i}}$.

2ำ Passo: Determinação do estoque inicial previsto

Para a determinação dos pedidos de FMItem faz-se necessário calcular o estoque no início do período. Com base no estoque real (Estoque FMItem no dia 20 do mês corrente), no saldo de pedidos confirmados a receber do fornecedor de FM, na antecipação de atendimento de pedidos do mês posterior (podem ter sido parcialmente registrados para reservar capacidade) e no saldo de pedidos de produto acabado do mês corrente a programar, obtém-se o estoque inicial a partir do qual os cenários simulados serão construídos. Este processo é mostrado na Figura 2.

3- Passo: Simulação do pedido prévio mensal

Nessa fase, utilizando as distribuições de probabilidade de acerto da previsão de vendas - PV (distribuição empírica) e distribuição do mix de FMItem (distribuição empírica) dentro do grupo de vendas, o sistema retorna o pedido ideal

Figura 2: Diagrama de determinação do estoque inicial.

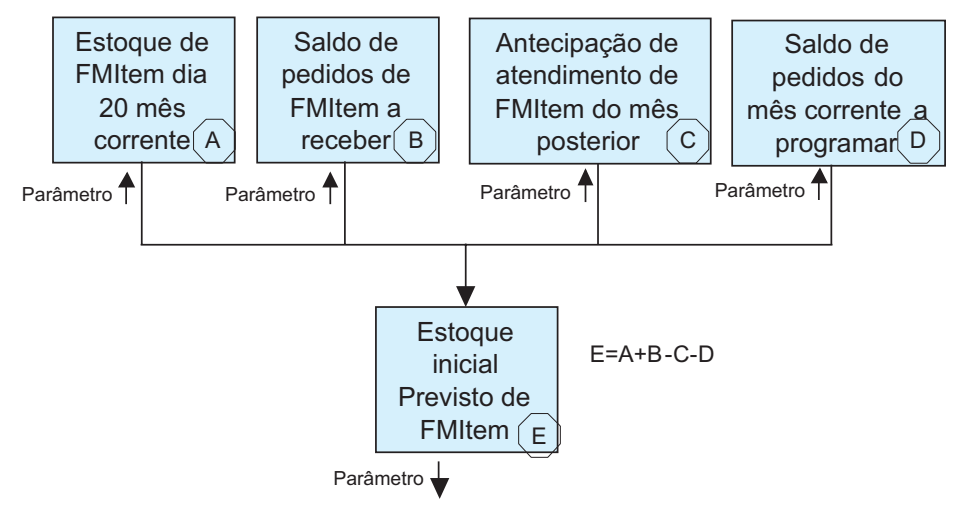

Freqüência: Mensal 
para cada FMItem avaliando os parâmetros de estoque inicial e de segurança calculados anteriormente. $\mathrm{O}$ resultado da simulação é obtido tendo como base diversas observações do sistema, cada uma delas usando amostragens das variáveis aleatórias consideradas. Para cada observação realizada é gerado aleatoriamente um erro para a previsão das quantidades estabelecidas para cada $\mathrm{GV}$, de forma a simular os erros de previsão com base nos erros históricos cometidos. De forma análoga, é feita uma geração aleatória da composição de cada $\mathrm{GV}$, baseada nas composições históricas de cada GV.

O pedido sugerido nessa etapa, ainda não contempla restrições de fornecimento (cota) e ajustes de níveis de prioridade de atendimento desejados. Isso será obtido na próxima fase de processamento das informações pelo sistema. A Figura 3 representa o processo.

\section{Passo: Simulação do pedido final}

Aqui é corrigido o pedido calculado na fase anterior com referência a cotas de fornecimento de FMItem, níveis de prioridade para alocação dessa cota e restrições de capacidade (nível máximo e mínimo - produção para estoque).

Como resultados são obtidos dados de pedido, estoque e nível de serviço no atendimento (médio, mínimo e máximo) do cenário desejado. Essas informações apoiarão o toma- dor de decisão na escolha do tamanho do pedido de cada FMItem. A Figura 4 apresenta o diagrama representativo do processo.

\section{modelo, apesar de simplificado em relação ao sistema estudado, apresentou resultados que retratam a realidade com boa qualidade.}

\section{PARÂMETROS DO SISTEMA}

Adicionalmente, foram definidos os demais parâmetros do sistema. Eles interferem nos resultados apresentados pelo modelo e são as variáveis disponíveis para que se possam construir cenários. Nos Quadros 1A e 1B destaca-se cada um deles com algumas recomendações a serem seguidas pelo tomador de decisões.

Tendo em vista a grande variabilidade de demanda apresentada por alguns FMItem durante a análise e tratamento dos dados, fez-se necessário estabelecer alguns limites de aplicabilidade do modelo, caso contrário os resultados poderiam diferir substancialmente da realidade. Escolheu-se a repetitividade e a variabilidade no histórico da demanda como condições limites. A primeira restrição, repetitividade, é um parâmetro de entrada do sistema. Para a restrição de variabilidade foi elaborado

Figura 3: Diagrama do processo de simulação do pedido prévio.

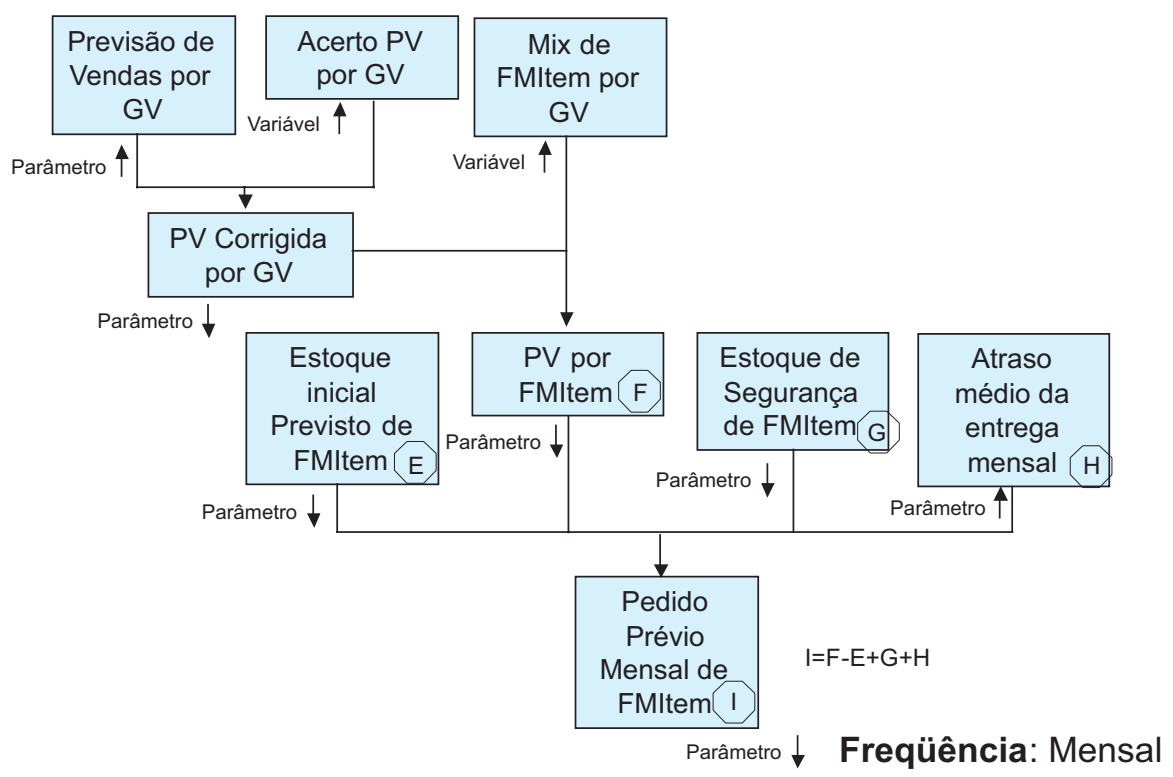


um teste (TDP - teste do desvio padrão) que é apresentado juntamente com os dados do resultado da simulação para apoiar e orientar o tomador de decisão sobre a adequação do modelo àquele histórico. Quanto maior a variabilidade, menor a adequação do modelo construído para aquele FMItem.

O sistema fornece as informações geradas pela simulação do processo que servirão de apoio para o tomador de decisão definir o pedido de FM ao fornecedor.

O Quadro 2 apresenta as informações fornecidas para cada cenário simulado.

Ressalta-se que as informações contidas nesse relatório devem ser utilizadas para apoiar a decisão e que informações e avaliações complementares devem ser efetuadas. Por isso as informações das quatro últimas linhas do Quadro 2 foram adicionadas aos resultados do processo de simulação para formar um relatório gerencial integrado. Essas informações são dados de entrada e foram simplesmente adicionadas aos respectivos resultados da simulação do processo de compra de cada FMItem.

\section{COLETA DE DADOS}

Nessa fase foram especificados e coletados os dados que apóiam o processo de tomada de decisão de compra e estoque do insumo FM. Foram eles:

- Demanda dos itens de FM (FMItem);

- Previsão de vendas (Por GV);

- Mix de FMItem por GV;
- Níveis de Estoque de FM, de cada FMItem, no final do mês;

- Lead time histórico dos pedidos de FM (pedidos colocados $\mathrm{x}$ atendimento realizado);

- Nível de Serviço desejado para o estoque de FMItem.

\section{ANÁLISE E TRATAMENTO DOS DADOS}

Após consolidação dos dados coletados e tratamento para adequação aos procedimentos exigidos durante a construção do modelo, foi desenvolvido um banco de dados composto por tabelas que devem ser continuamente alimentadas para possibilitar o processo de simulação que acontecerá periodicamente (a cada mês). O Quadro 3 apresenta as principais tabelas da base de dados.

\section{VERIFICAC̣ÃO E VALIDAC̣̃̃O DO MODELO}

O modelo foi verificado utilizando-se apenas um grupo de vendas e com uma distribuição empírica de freqüência que produzia resultados previsíveis, o que possibilitou a contraprova manual atestando a confiabilidade dos cálculos realizados.

O modelo foi validado através de testes para verificação de seu grau de aderência ao processo real com base nos meses de outubro, novembro e dezembro de 2004 e janeiro de 2005. O período foi escolhido por ter todos os dados históricos disponíveis para alimentação do modelo e comparação com o desempenho real da demanda de FM.

Figura 4: Diagrama do processo de simulação do pedido final.

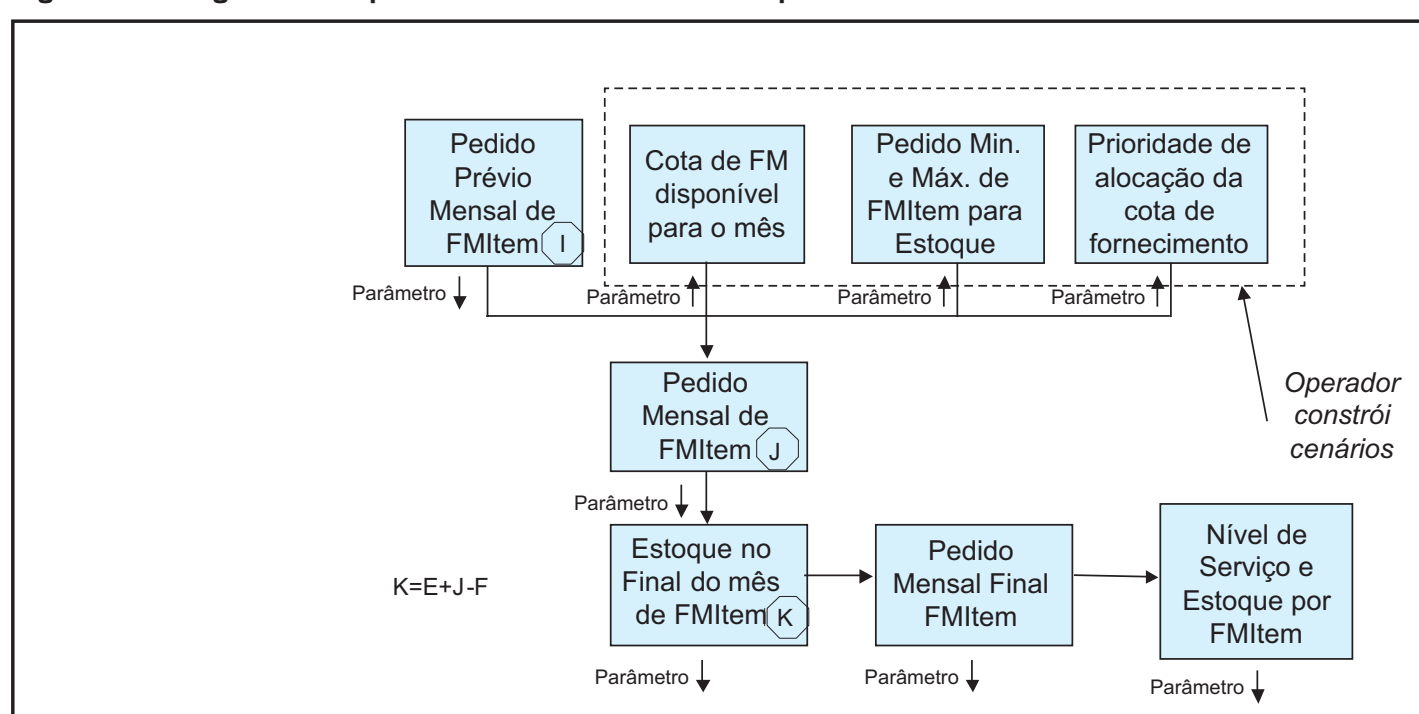

Freqüência: Mensal 
Quadro 1A: Parâmetros do sistema.

\begin{tabular}{|c|c|c|}
\hline ITEM & DESCRIÇÃO DO ITEM & OBSERVAÇÕES \\
\hline Prioridade & $\begin{array}{l}\text { Critério de peso/importância para ajustar a cota de aquisição } \\
\text { aos diversos FMltem. } \\
\text { Quanto maior o peso, maior a importância relativa do item }\end{array}$ & Escala de 1 a 4. \\
\hline Pedido min & $\begin{array}{l}\text { Utilizado para forçar um pedido de compra de FMItem mesmo } \\
\text { que a previsão de vendas não indique o consumo. } \\
\text { Está relacionada à política de produção para utilização da } \\
\text { capacidade instalada }\end{array}$ & Em toneladas \\
\hline Pedido max & $\begin{array}{l}\text { Utilizado para limitar um pedido de compra de FMltem mesmo } \\
\text { que a previsão de vendas indique um consumo superior. } \\
\text { Está relacionada à política de produção e às restrições da } \\
\text { capacidade instalada }\end{array}$ & Em toneladas \\
\hline Dias de cobertura & $\begin{array}{l}\text { Parâmetro utilizado para aumentar o estoque de segurança } \\
\text { em função do cronograma de laminação de FM e flutuações de } \\
\text { demanda acentuadas }\end{array}$ & Número de dias \\
\hline Seg lead time & $\begin{array}{l}\text { Coeficiente de segurança do lead time. Utilizado para definir } \\
\text { o índice de acerto (baseado no histórico de fornecimentos) } \\
\text { que se pretende obter para o FMltem no que diz respeito } \\
\text { ao prazo de entrega praticado pelo fornecedor. Influencia no } \\
\text { cálculo do estoque de segurança. Quanto maior o coeficiente, } \\
\text { maior o nível de estoque de segurança recomendado. }\end{array}$ & $\begin{array}{l}0,0 \text { - baixo } \\
0,5 \text { - médio } \\
1,0 \text { - importante } \\
2,0 \text { - elevado } \\
\text { Obs: recomenda-se o mesmo } \\
\text { critério do SEG DEMANDA }\end{array}$ \\
\hline Seg demanda & $\begin{array}{l}\text { Coeficiente de Segurança da Demanda. Utilizado para definir } \\
\text { o índice de acerto (baseado no histórico de fornecimentos] } \\
\text { que se pretende obter para o FMltem no que diz respeito ao } \\
\text { volume histórico consumido. Influencia no cálculo do estoque } \\
\text { de segurança. Quanto maior o coeficiente, maior o nível de } \\
\text { estoque de segurança recomendado. }\end{array}$ & $\begin{array}{l}0,0 \text { - baixo } \\
0,5 \text { - médio } \\
\text { 1,0 - importante } \\
2,0 \text { - elevado } \\
\text { Obs: recomenda-se o mesmo } \\
\text { critério do SEG LEAD TIME }\end{array}$ \\
\hline
\end{tabular}

Quadro 1B: Parâmetros do sistema (continuação).

\begin{tabular}{|c|c|c|}
\hline ITEM & DESCRIÇÃO DO ITEM & OBSERVAÇÕES \\
\hline $\begin{array}{l}\text { Número de } \\
\text { observações }\end{array}$ & $\begin{array}{l}\text { Refere-se à quantidade de amostras utilizadas nos cálculos } \\
\text { da simulação para obter as respostas desejadas }\end{array}$ & $\begin{array}{l}\text { Recomenda-se que não se use } \\
\text { menos de } 200\end{array}$ \\
\hline Repetitividade & $\begin{array}{l}\text { Número mínimo de meses em que o FMltem aparece no } \\
\text { histórico de consumo. Trata-se de restrição para inclusão do } \\
\text { item na simulação de pedido }\end{array}$ & $\begin{array}{l}\text { Recomendam-se } 8 \text { presenças } \\
\text { em } 12 \text { meses }\end{array}$ \\
\hline Uso de cota & $\begin{array}{l}\text { Instrução para autorização de ajustes no pedido de compra } \\
\text { em função de uma eventual cota de aquisição junto ao } \\
\text { fornecedor }\end{array}$ & $\begin{array}{l}\text { Padrão: (NÃO) não utiliza } \\
\text { restrição de cota. } \\
\text { Para utilizar restrição de cota } \\
\text { alterar o parâmetro para } \\
\text { (SIM] }\end{array}$ \\
\hline Cota de FM (t) & Volume total em toneladas da cota de aquisição & \\
\hline Uso do GV & $\begin{array}{l}\text { Indica quais Grupos de Vendas devem ser utilizados. } \\
\text { É utilizado para eliminar GVs de produtos acessórios que não } \\
\text { têm o FM como insumo. Ex: postes para cercas. }\end{array}$ & (SIM) ou (NÃO) \\
\hline
\end{tabular}


Os dados apresentados a seguir foram obtidos utilizando dados históricos referentes aos seis meses anteriores ao mês que se pretende planejar as compras e estoques de FM (dados móveis). Adicionalmente, foram considerados os seguintes parâmetros:
- Número de observações: 200;

- Sem restrição de cota de aquisição;

- Coeficientes de segurança da demanda e do lead time de fornecimento bem como o pedido mínimo e máximo e dias de cobertura de cada FMItem previamente

Quadro 2: Informações de saída do sistema.

\begin{tabular}{|c|c|}
\hline ITEM & DESCRIÇÃO \\
\hline TDP & $\begin{array}{l}\text { Teste do Desvio Padrão. Indica a variabilidade histórica da demanda do item. Escala de } 0 \text { a } 1 . \\
\text { Quanto maior o TDP menor a variabilidade. }\end{array}$ \\
\hline Rep & Número de meses que o FMltem apareceu no histórico considerado \\
\hline Est Ini & Estoque no início do mês \\
\hline Est Seg & Estoque de segurança calculado com base nos parâmetros indicados \\
\hline PV Min & Previsão de venda mínima simulada \\
\hline PV Média & Previsão de venda média simulada \\
\hline PV Max & Previsão de venda máxima simulada \\
\hline Ped Prev & $\begin{array}{l}\text { Pedido sugerido para recompor o estoque de segurança com base na PV Média antes da correção } \\
\text { da cota de aquisição (se houver) }\end{array}$ \\
\hline PcorMin & $\begin{array}{l}\text { Pedido sugerido para recompor o estoque de segurança com base na PV Min depois da correção da } \\
\text { cota de aquisição (se houver) }\end{array}$ \\
\hline PcorMed & $\begin{array}{l}\text { Pedido sugerido para recompor o estoque de segurança com base na PV Média depois da correção } \\
\text { da cota de aquisição (se houver) }\end{array}$ \\
\hline PcorMax & $\begin{array}{l}\text { Pedido sugerido para recompor o estoque de segurança com base na PV Max depois da correção da } \\
\text { cota de aquisição (se houver) }\end{array}$ \\
\hline EstFinal & Estoque no final do mês previsto para o FMltem \\
\hline Ped Reg & Pedidos de FMltem já registrados para o mês seguinte [alvo] \\
\hline $\mathrm{CX}+1$ & Volume em toneladas da carteira de arame já registradas para o mês $X+1$ \\
\hline $\mathrm{CX}+2$ & Volume em toneladas da carteira de arame já registradas para o mês $X+2$ \\
\hline $\mathrm{CX}+3$ & Volume em toneladas da carteira de arame já registradas para o mês $X+3$ \\
\hline
\end{tabular}

Quadro 3: Tabelas da base de dados.

\begin{tabular}{|l|l|}
\hline \multicolumn{1}{|c|}{ NOME DA TABELA } & \multicolumn{1}{c|}{ DESCRIçÃ̃o } \\
\hline tb_acerto_previsao_gv & $\begin{array}{l}\text { Contém informações comparativas da previsão de vendas por GV com } \\
\text { as vendas reais efetuadas a cada mês }\end{array}$ \\
\hline tb_distribuicao_demanda_mensal & $\begin{array}{l}\text { Contém informações do histórico de consumo mensal (com base na } \\
\text { carteira de vendas) dos FMltem }\end{array}$ \\
\hline tb_Estoque_Inicial_FM & $\begin{array}{l}\text { Tabela de entrada de dados relativos à situação de estoques na data } \\
\text { em que o sistema está sendo utilizado (mês corrente) }\end{array}$ \\
\hline tb_leadtime_pedidos_fm_histórico & $\begin{array}{l}\text { Registra o histórico de atendimento dos pedidos de FM pelo } \\
\text { fornecedor }\end{array}$ \\
\hline tb_percentual_histórico_fm_gv & $\begin{array}{l}\text { Contém os registros do percentual de participação de cada FMltem } \\
\text { num determinado GV }\end{array}$ \\
\hline tb_previsao_vendas_gv & $\begin{array}{l}\text { Registra a previsão de vendas por grupo de vendas para o mês } \\
\text { subseqüente }\end{array}$ \\
\hline
\end{tabular}


especificados, foram mantidos fixos em todos os experimentos;

- Informações obtidas utilizando a recomendação de pedido médio indicado pela simulação do processo;

- Dados multiplicados por uma constante para preservar o sigilo das informações da empresa.

A Tabela 1, traz os resultados do processo de validação do modelo de simulação. O significado de cada item é descrito a seguir.

\section{RESULTADOS}

O modelo utilizou técnicas de modelagem matemática que levam em consideração parâmetros estabelecidos por especialistas visando obter um resultado melhor do que se poderia obter utilizando apenas estimativas e/ou métodos muito simplificados que não retratam a dinâmica do processo estudado.

A seguir é apresentado o detalhamento dos resultados obtidos no período em que o modelo foi validado: meses de outubro, novembro e dezembro de 2004 e janeiro de 2005.

Média de FMItem simulados por mês: $46 \%$.

Em $93 \%$ dos itens a política de aquisição sugerida pelo pedido médio do sistema satisfez a demanda real.

$\mathrm{O}$ volume médio de vendas não atendido por falta de FM foi de $1,9 \%$ das vendas (itens que apresentaram ruptura).

Dentre os FMItem que apresentaram ruptura, um número bastante reduzido representou $45 \%$ das rupturas e devem ter seus históricos de demanda analisados com maior profundidade para entender os fatores que interferiram nesse processo.
Em janeiro, houve ruptura de estoque acentuada (em volume) em dois FMItem que juntos representaram $68 \%$ do volume faltante no mês. Nesses casos, a situação foi analisada e o crescimento exagerado das vendas em relação ao histórico de dados gerou o problema. Neles, a base de dados histórica não representou bem a realidade e, por isso, o estoque de segurança não foi capaz de proteger a produção e ocorreu assim falta de FM para atender aos pedidos registrados (ruptura).

O estoque real de fio máquina no final do mês ficou, em média, $14 \%$ acima do volume de estoque previsto, indicando que houve variação para baixo das vendas de alguns itens de grande demanda em relação ao histórico de vendas. Os FMItem que apresentaram estoques elevados em relação ao estoque projetado foram analisados e, nesses casos, a redução drástica nos volumes de venda comprometeu a previsão do modelo.

Perfil de demanda dos FMItem segundo o Teste do Desvio Padrão - TDP:

$$
\begin{aligned}
& \text { TDP }=1,00(56 \% \text { dos itens }) \\
& \text { TDP }=0,75(16 \% \text { dos itens }) ; \\
& \text { TDP }=0,50(19 \% \text { dos itens }) ; \\
& \text { TDP }=0,25(7 \% \text { dos itens }) .
\end{aligned}
$$

A empresa permanece utilizando a ferramenta para apoiar seu processo de tomada de decisão de aquisição do insumo FM.

Especialistas continuam envolvidos no processo e validam a ferramenta.

O modelo, apesar de simplificado em relação ao sistema

\begin{tabular}{|c|c|c|c|c|c|c|c|c|c|c|c|c|c|c|c|}
\hline Ano & Mês & itens & ESeg & Elni & PMed & EProj & PVMin & PVMed & PVMax & Venda & ERealMed & RMed & Prup & MedPRupMed & TRMed \\
\hline 2004 & 10 & 228 & 93 & 90 & 247 & 187 & 152 & 242 & 389 & 189 & 156 & 23 & $10 \%$ & $4,54 \%$ & 9 \\
\hline 2004 & 11 & 223 & 92 & 123 & 210 & 190 & 148 & 236 & 388 & 161 & 173 & 12 & $5 \%$ & $0,56 \%$ & 1 \\
\hline 2004 & 12 & 216 & 80 & 101 & 194 & 166 & 136 & 209 & 336 & 145 & 155 & 9 & $4 \%$ & $2,91 \%$ & 4 \\
\hline 2005 & 1 & 198 & 79 & 97 & 164 & 164 & 112 & 177 & 296 & 164 & 123 & 18 & $9 \%$ & $15,32 \%$ & 25 \\
\hline
\end{tabular}
estudado, apresentou resultados que retratam a realidade com boa qualidade.

Tabela 1: Resultados do processo de validação do modelo de simulação.

\section{Legenda:}

Itens

Eseg

Eini

Pmed

Eproj

PVMin

PVMed

PVMax

Venda

ERealMed

Rmed

PRup

MedPRupMed

TRMed
Número de FMltem simulados;

Estoque de segurança total indicado para os itens;

Estoque no início do mês:

Pedido médio sugerido pelo sistema;

Estoque projetado para o final do mês;

Previsão de vendas mínima simulada;

Previsão de vendas média simulada;

Previsão de vendas máxima simulada;

Vendas reais apuradas no mês:

Estoque real apurado para os itens no final do mês considerando colocação do Pedido Médio;

Número de rupturas de estoque dentre os FMltem simulados considerando a sugestão de Pmed;

Percentual de rupturas de estoque dentre os FMltem simulados;

Percentual médio do volume vendido que faltou para ser atendido;

Tonelagem total não atendida. 


\section{CONCLUSÕES}

As premissas do estudo foram consideradas adequadas e é possível afirmar que o comportamento histórico das previsões de venda de produtos acabados e o respectivo mix de produtos necessários à produção, adicionados a parâmetros e restrições de produção, podem determinar, com boa precisão, o planejamento de compra de insumos. Além disso, uma ferramenta simples baseada nesses princípios produziu melhorias substanciais nos resultados do processo de tomada de decisão.

As exceções de adequação apontadas pela ruptura no estoque indicaram inadequação do histórico de vendas e fornecimento à realidade do mês em que se projetaram as vendas, o que é previsível num projeto de simulação que usa dados probabilísticos.
- Verificação da possibilidade de aumento do histórico de dados que represente a realidade atual. Históricos maiores que seis meses aumentariam a confiabilidade das aproximações estatísticas. Fez-se o teste no mês de janeiro de 2005 com 12 meses de histórico e obteve-se um menor número de itens apresentando rupturas, bem como um aumento expressivo no número de FMItem simulado.

- Considerações adicionais devem ser realizadas durante a tomada de decisão no caso de produtos que podem sofrer elevação de preços. Nessa situação, é sabido que o forte crescimento das vendas reduz a representatividade do histórico, base da análise do sistema. A previsão desse impacto deve ser avaliada e acrescentada aos resultados do modelo.

- É necessária a revisão contínua e periódica dos parâmetros de estoque com base nas alterações de processo, cronograma de laminação e restrições de capacidade de produção.

- Durante a alimentação dos dados históricos no sistema, deve-se garantir que alterações de processo estejam contempladas. Aproveitar base de dados de meses anteriores somente adicionando informações recentes pode gerar erro.

- TDP menor que 1 indica que o tomador de decisão deve tomar cuidado extra ao definir o pedido de compra de FMItem, já que a variação de demanda se mostrou acentuada no histórico utilizado.

- Calcular custos de manutenção de estoques e estimar custos de vendas postergadas e vendas perdidas para ajustar de forma quantitativa (e não subjetiva) os parâmetros de estoque de segurança.

$\mathrm{O}$ modelo pode ser considerado adequado a apoiar o processo de decisão de compra de FM na empresa tendo em vista o grande número de FMItem cujo histórico de demanda valida o modelo construído para determinação do tamanho do pedido de compra. 


\section{- Referências Bibliográficas}

ARNOLD, J. R. T. Administração de Materiais: uma introdução. São Paulo: Atlas, 521 p., 1999.

BALLOU, R. H. Gerenciamento da Cadeia de Suprimentos: planejamento, organização e logística empresarial. 4. ed. Porto Alegre: Bookman, 532 p., 2001.

BARTEZZGHI, E.; VERGANTI, R.; ZOTTERI, $G$. Measuring the impact of asymmetric demand distributions on inventories. International Journal of Production Economics, v. 60-61, p. 395-404, 1999.

CORRÊA, H. L.; GIANESI, I. G. N.; CAON, M. Planejamento, Programação e Controle da Produção com MRPII / ERP: Conceitos, Uso e Implantação. 4. ed. São Paulo: Atlas, 452 p., 2001.

COUGHLAN P.; COGHLAN D. Action research for operations management. International Journal of Operations \& Production Management, v. 22, n. 2 , p. 220-240, 2002

HAUTANIEMI, P.; PIRTTILÄ, T. The choice of replenishment policies in an MRP environment. International Journal of Production Economics, v. 59, p. 85-92, 1999.
HILLIER, F.; LIEBERMAN, G. Introduction to Operations Research. 8. ed. New York: McGraw Hill, 1061 p., 2005.

JACOBS, F. R.; WHYBARK, D. C. A comparison of Reorder Point and Material Requirements Planning Inventory Control Logic. Decision Sciences, v. 23, n. 2, p. 332 343, 1992.

KREVER, M.; WUNDERINK, S.; DEKKER, R.; SCHORR, B. Inventory control based on advanced probability theory, an application. European Journal of Operational Research, v. 162, n. 2, p. 342-358, 2003.

KRUPP, J. A. G. Safety stock management. Production and Inventory Management Journal, v. 38, n. 3, p. 11-18, 1997.

KRUPP, J. A. G. Integrating Kanban and MRP to reduce lead time. Production and Inventory Management Journal, v. 43 p. 78-92, 2002.

LENARD, J. D.; ROY, B. Multi-Item Inventory Control: A Multicriteria View. European Journal of Operational Research, v. 87, p. $685-692,1995$

PANG, W. K.; LEUNG, P. K.; HUANG, W. K.; LIU, W. On interval estimation of the coefficient of variation for the threeparameter Weibull, Lognormal and Gamma distribution: a simulation-based approach. European Journal of Operational Research, v. 164, n. 2, p. 367-377, 2003.

PARTOVI, F. Y.; ANANDARAJAN, M. Classifying inventory using an artificial neural network approach. Computers \& Industrial Engineering, v. 41, p. 389404, 2002.

PEIXOTO, E. C. Planejamento de pedidos de compra e estoques de insumos a partir da previsão agregada de vendas e histórico de demanda utilizando simulação. Dissertação de Mestrado. Departamento de Engenharia de Produção, Universidade Federal de Minas Gerais, 70 p., 2006.

PETROVIC, R.; PETROVIC, D. Multicriteria ranking of inventory replenishment policies in the presence of uncertainty in customer demand. International Journal of Production Economics, v. 71, p. 439-446, 2001.

PLATT, D.; ROBINSON, L. W.; FREUND, R. B. Tractable $(Q, R)$ Heuristic Models for Constrained Service Levels. Management Science, v. 43, n. 7, p. 951, 1997.
PRITSKER, A. A. B. Simulation: The Premier Technique of Industria Engineering. Industrial Engineering. v. 24, n. 7, p. 25-26, 1992.

RAMANATHAN, R. ABC inventory classification with multiple-criteria using weighted linear optimization. Computers \& Operations Research, v. 33, n. 3, p. 695$700,2004$.

SANTOS, A. M.; RODRIGUES, I. A. Desenvolvimento de um sistema de controle de estoques para apoio à tomada de decisão num ambiente de materiais com diferentes padrões de demanda. In: XXV Encontro Nacional de Engenharia de Produção - ENEGEP. Anais, Porto Alegre, 2005.

SHANNON, R. E. Systems Simulation: The Art and the Science. New Jersey: PrenticeHall, 387 p., 1975.

YEH, Q. J.; CHANG, T. P.; CHANG, H. C. An inventory control model with Gamma distribution. Microelectronics and Reliability, v. 37 , n. 8, p. 1197-1201, 1997.

\section{- Sobre os autores}

\section{Eduardo Carrara Peixoto}

Belgo Bekaert Nordeste S.A.

Assistente Técnico, M.Sc.

End.: Rua Oscar de Freitas, 121 - Casa 40 - Feira de Santana - BA - 44055-170

Tel.: (75) 3616-6417

E-mail: educarrara@bol.com.br

\section{Luiz Ricardo Pinto}

Universidade Federal de Minas Gerais

Professor Adjunto, D.Sc.

End.: Av. Antônio Carlos, 6627 - Pampulha - Belo Horizonte - MG - 31270-901

Tel.: 3499-4901 Fax: 3499-4888

E-mail: luiz@dep.ufmg.br 